\title{
Multilevel modular mesocrystalline organization in red coral
}

\section{Daniel Vielzeuf, ${ }^{1, *}$ Nicole Floquet, ${ }^{1}$ Dominique Chatain, ${ }^{1}$ Françoise Bonneté, ${ }^{1}$ DANIEL FERry, ${ }^{1}$ JOAQUim GARRABOU, ${ }^{2}$ AND EDWARD M. STOLPER ${ }^{3}$}

\author{
${ }^{1}$ Centre Interdisciplinaire de Nanoscience de Marseille, CNRS-Aix-Marseille University, 13288, Marseille, France \\ ${ }^{2}$ Institut de Ciències del Mar, Passeig Maritim de la Barceloneta 37-49, 08003 Barcelona, Spain \\ ${ }^{3}$ Division of Geological and Planetary Sciences, California Institute of Technology, MC 170-25, 1200 East California Boulevard, Pasadena, \\ California 91125, U.S.A.
}

\begin{abstract}
Biominerals can achieve complex shapes as aggregates of crystalline building blocks. In the red coral skeleton, we observe that these building blocks are arranged into eight hierarchical levels of similarly (but not identically) oriented modules. The modules in each hierarchical level assemble into larger units that comprise the next higher level of the hierarchy, and consist themselves of smaller, oriented modules. EBSD and TEM studies show that the degree of crystallographic misorientation between the building blocks decreases with decreasing module size. We observe this organization down to a few nanometers. Thus, the transition from imperfect crystallographic order at millimeter scale to nearly perfect single crystalline domains at nanometer scale is progressive. The concept of "mesocrystal" involves the three-dimensional crystallographic organization of nanoparticles into a highly ordered mesostructure. We add to this concept the notion of "multilevel modularity." This modularity has potential implications for the origin of complex biomineral shapes in nature. A multilevel modular organization with small intermodular misorientations combines a simple construction scheme, ruled by crystallographic laws, with the possibility of complex shapes. If the observations we have made on red coral extend to other biominerals, long-range crystallographic order and interfaces at all scales may be key to how some biominerals achieve complex shapes adapted to the environment in which they grow.
\end{abstract}

Keywords: Biomineral, mesocrystal, crystallography, calcite, EBSD, hierarchical organization, modularity, Corallium rubrum, complex shape

\section{INTRODUCTION}

Biominerals often display morphological, chemical, and crystallographic patterns at length scales ranging from the nano- to the macroscale (Lowenstam and Weiner 1989; Mann 2001). As such, they correspond to the definition of "complex systems" (Grimm et al. 2005). Either individually or in combination with each other, these patterns can provide information on the mechanisms that produce complex structures. An important feature of biominerals is that they can achieve "beautifully sculpted" shapes (Weiner et al. 2005) using crystal symmetry-dependent blocks, but it is unclear how these shapes are formed from such building blocks. One possibility is that a disordered amorphous precursor phase that can be "molded into any shape" (Weiner et al. 2005) plays an important role in generating a wide variety of forms (Pecher et al. 2009; Weiner et al. 2005). Another is that complex shapes can emerge from particular arrangements of crystalline units during growth (Towe 2006). In this article, we demonstrate that red coral (Corallium rubrum) acts as an astute crystallographer, assembling its skeleton as a delicate arrangement of a hierarchy of crystals with well-defined orientations relative to their near- and far-field neighbors.

\footnotetext{
*E-mail: vielzeuf@cinam.univ-mrs.fr
}

\section{EXPERIMENTAL METHODS}

Electron backscatter diffraction (EBSD) using a SEM enables quantitative measurements of the crystallographic orientation of crystal domains as small as $200 \mathrm{~nm}$ (Prior et al. 1999), and the calculation of misorientation axes and angles between any two data points. EBSD patterns were obtained at Caltech on a LEO 1550VP SEM equipped with a HKL technology "channel 5" EBSD system using an accelerating voltage of $20 \mathrm{kV}$, a probe current of $2 \mathrm{nA}$, and a working distance of $14 \mathrm{~mm}$. Samples were prepared by conventional polishing using diamond paste with grit sizes down to 1 or $1 / 4 \mu \mathrm{m}$, followed by a final polish with colloidal silica. Collection times of $\sim 0.3 \mathrm{~s}$ under the SEM beam were sufficient to generate good quality EBSD patterns for the Mg-calcite red coral skeleton. A $200 \times 100$ grid with $4 \mu \mathrm{m}$ spacing between points generated an orientation map in $<2 \mathrm{~h}$. In the orientation map, each pixel corresponds to a crystallographic orientation characterized by three Euler angles $(\varphi 1, \Phi, \varphi 2)$ transformed into a RGB color code using the relations red $=255 \varphi_{1} / 180$, green $=255 \Phi / 180$, blue $=255 \varphi_{2} / 180$. The lattice orientations of the Mg-calcite crystals making up the red coral skeleton (Grillo et al. 1993) were determined with a lateral diameter of the diffracting volume of $\sim 200 \mathrm{~nm}$ and an absolute angular resolution of $\pm 0.5^{\circ}$. EBSD patterns with a mean angular uncertainty $\geq 1^{\circ}$ were discarded and plotted as white dots on the map; on the orientation map, $42.6 \%$ of the points were indexed. Stereographic projections (upper hemisphere) of $\{\mathrm{hkil}\}$ planes and the corresponding pole density/contour diagrams were automatically generated.

A SEM equipped with a forescatter detector generates images of texture of polycrystalline samples with a submicrometer spatial resolution (Prior et al. 1999). SEM orientation contrast (OC) imaging is based on signal contrast between materials with differences in crystallographic orientations. Sharp contrast changes correspond to sudden changes in crystallographic orientations. This technique detects small changes in orientation $\left(<0.5^{\circ}\right)$ unseen by EBSD (Prior et al. 1999). It is also a high spatial resolution technique since domains about $0.25 \mu \mathrm{m}$ wide can be resolved with a field emission source. OC images are not quantitative (in 
contrast to EBSD), but they do allow the characterization of small crystallographic changes in polycrystalline materials.

Other more widely used techniques (SEM and TEM) and the provenance of the biological material are described in Vielzeuf et al. (2008). Results presented here are based on the study of about 20 different samples of red coral.

\section{RESULTS}

In this section, the crystallographic structure of the red coral skeleton is presented from the largest to the smallest unit. In the figures, features of interest are labeled (e.g., "he" for herringbone unit, etc.); in the text, they are referred to by the figure number followed by the label (e.g., Fig. 1b [he]).

\section{Herringbone units and strips $\left(10^{-2}-10^{-3} \mathrm{~m}\right)$}

Observation of polished surfaces of red coral cut perpendicular to its main axis under a reflected light microscope shows a regular alternation of a few millimeters long, darker and brighter strips (Figs. 1a and $1 \mathrm{~b}[d s, b s]$ ). A dark strip indicates that the optical axes of the $\mathrm{Mg}$-calcite crystals that compose the skeleton are parallel or perpendicular to the axes of the polarizer or analyzer, while any other orientation generates bright strips. A rotation of $45^{\circ}$ reverses the pattern. The alternation of bright and dark strips in a radial arrangement is shown in Figure 1a. It is important to note that crystals in a single strip are, from a crystallographic point of view, almost similarly oriented over distances of a few millimeters. The coupling of a dark and a bright strip forms a composite crystallographic superstructure that we refer to as the herringbone unit (Figs. 1b [he] and 1c). Some herringbone units reach the crenulated rim of the skeleton (Fig. 1a), and each of these units coincides with a concave outward portion of the rim (Fig. 1a $[\mathrm{cr}]$ ); the wavy pattern at the rim is the expression in cross section of longitudinal grooves running along the skeleton (Grillo et al. 1993; Vielzeuf et al. 2008). The herringbone units display a roughly constant width, ca. 200-300 $\mu \mathrm{m}$ (Figs. 1a and 1b). Thus, the increase in diameter of the skeleton is accommodated not by an increasing width of the herringbone units but by an increasing number of units (Fig. 1a). New units appear at macroscopic dislocations (Figs. 1a and 1b [di]).

Figure $2 b$ shows an EBSD orientation map with three herringbone units, superposed upon a reflected light microphotograph (Fig. 2a). The EBSD study was performed on a different sample than those shown in Figure 1; however, as indicated by the scale bars, the width of the EBSD map is about twice the width of Figure 1c. The orientation map shows the alternation of blue and pink bands coinciding with the bright and dark strips of the photograph, respectively. Pole figures (Figs. 2c and 2d) indicate that the $c$ axes of the crystals of $\mathrm{Mg}$-calcite are almost parallel to the plane of observation XY (tilt $\left.<\sim 6^{\circ}\right)$ in both types of strips. However, most importantly, their orientations in that plane systematically differ: in the blue strips, the $c$ axes are oriented $\sim-22^{\circ}$ (relative to the Y axis shown in Fig. 2a), while they are oriented $\sim+22^{\circ}$ in the pink strips. The histogram of the crystallographic orientation of the $c$ axes in the XY plane in the blue strips (Fig. 2e) shows a unimodal distribution and a peak width at half height of $\sim 30^{\circ}$. The distribution for the pink strips is bimodal (Fig. 2e), but with a comparable peak width at half height if it is treated as a unimodal distribution. We do not ascribe important significance to this bimodality since it could be related to statistical bias.

\section{Spindles $\left(\mathbf{1 0}^{-4} \mathrm{~m}\right)$}

Observation at higher magnification indicates that the bright/ dark strips seen in reflected light are not perfectly homogeneous, but are composed of elongated and irregular spindle-shape sub units (Fig. 1b). These spindles are 100 to $300 \mu \mathrm{m}$ long and 10 to $50 \mu \mathrm{m}$ wide; some of them are contoured in Figure 1c [sp]. The angle between spindles in the bright and dark strips is in general close to $45^{\circ}$, but shows a large dispersion of $\pm 20^{\circ}$. It is this arrangement that results in the visually obvious herringbone pattern in Figure 1c. The combination of EBSD map (Fig. 2b), pole figures (Figs. 2c and 2d), and reflected light images show that the elongation of the spindles is more or less parallel to the $c$ optical axis of the crystals. Histograms of distribution of crystallographic orientations can be retrieved from zones corresponding to a single spindle (Figs. 2b and 2e). For a given set of EBSD data, the histogram of orientations of the $c$ axes of a strip is obviously the external envelope of the local histograms of the spindles that constitute the strip. The histogram for a single spindle displays a significantly lower spread of orientations than the strip as a whole (Fig. 2e) and a remarkable similarity of crystallographic orientation with a peak width at half height of only $\sim 15^{\circ}$, i.e., about half that observed for the strip as a whole. Normalization of the data to generate identical areas under the strip or spindle distribution curves does not affect the width at half height of the distributions. Other EBSD maps (not shown here) performed on the same sample with a better spatial resolution $(2$ and $1 \mu \mathrm{m}$ spacing between points) confirm this hierarchy of statistic distributions. Furthermore, the OC image of spindles shown in Figure $1 \mathrm{~d}$ is consistent with this observation: dark and bright lineations (emphasized by yellow lines in Fig. 1d) indicate a preferential crystallographic orientation within each spindle.

We noted earlier that the surface of the red coral skeleton is crenulated and shows a wavy pattern ( $300 \mu \mathrm{m}$ wavelength). The surface of each wave is not smooth but, in a self-similar fashion, made of numerous tree-like smaller $(\sim 30 \mu \mathrm{m})$ microprotuberances (Grillo et al. 1993; Vielzeuf et al. 2008). Similarity of orientation and size, together with other EBSD observations (not shown here) suggest that microprotuberances are the surface expression of the spindles.

\section{Lozenges $\left(10^{-5} \mathrm{~m}\right)$}

OC images at higher magnification (Fig. 1e) show that spindles are not single crystals. Instead, they are made of $5 \mu \mathrm{m}$ wide, $10 \mu \mathrm{m}$ long lozenges (Fig. 1e [lo]) separated by narrow, dark, porous zones (Fig. 1e [po]). In spite of being made of separate sub-units, the crystallographic orientation within each spindle remains nearly constant as indicated by the alignment of the long axis of the lozenges and the parallelism of dark/bright elongated bands (Fig. 1e [ba]). In both the spindles and the lozenges, EBSD showed that the long axes of these bands coincide with the $c$ axes of calcite crystals (Figs. 1d and 1e $[c]$ ).

\section{Fibers $\left(10^{-6} \mathrm{~m}\right)$}

The alternation of dark and bright elongated bands $(<1 \mu \mathrm{m}$ wide, $5-10 \mu \mathrm{m}$ long) in the lozenges (Fig. 1e $[b a]$ ) indicate that crystallographic misorientations still exist within each lozenge. However, these misorientations may be very small since minute differences can be detected by $\mathrm{OC}$ imaging $\left(<0.1^{\circ}\right.$ according to 

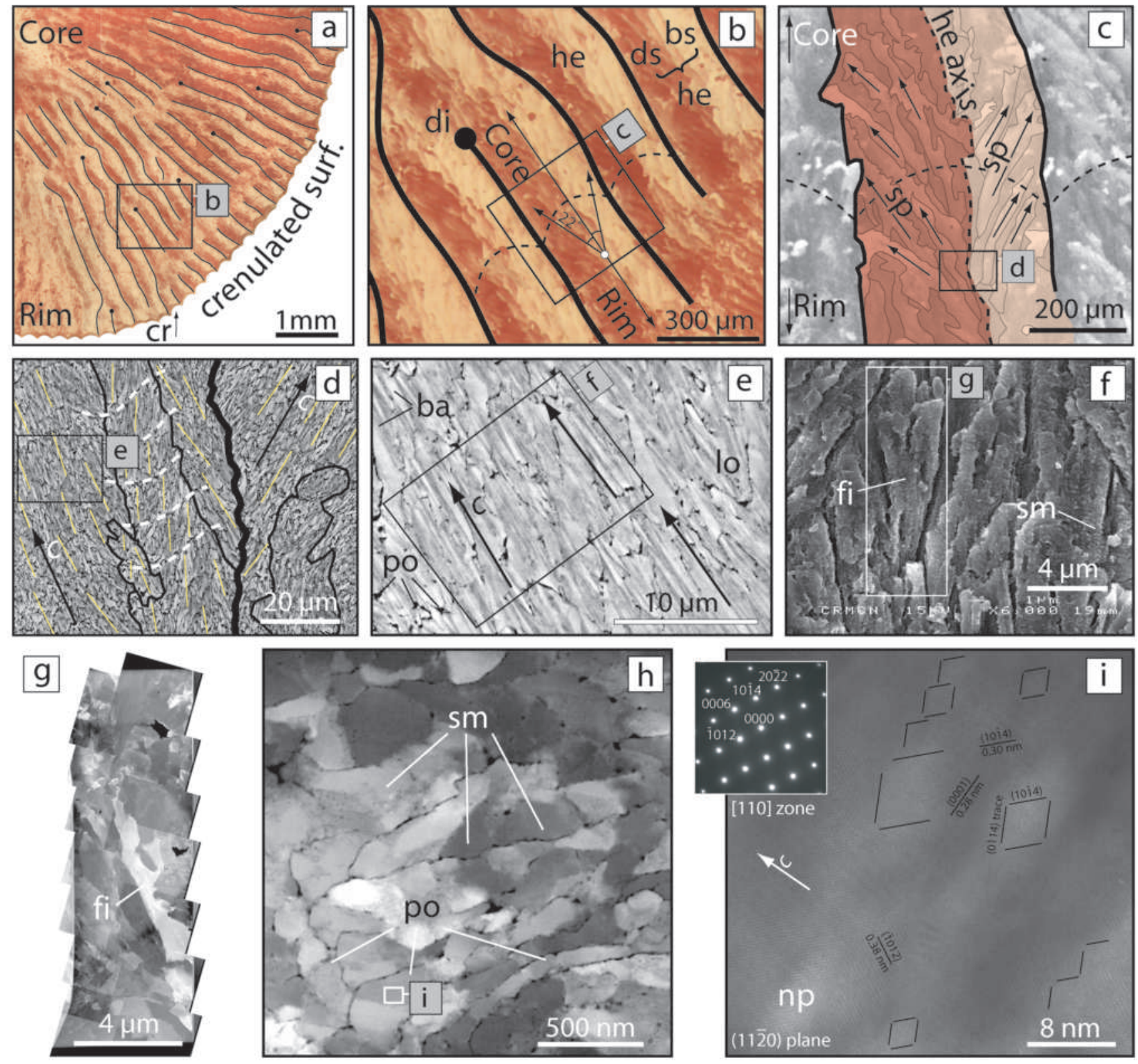

FIGURE 1. Crystallography of Corallium rubrum. (a) Mosaic photograph of a section of skeleton under reflected light microscope (polarized and analyzed light) with long-range crystallographic order and herringbone units. di: dislocation, $c r$ : trough on the wavy surface. (b) Enlargement of a. The herringbone units $(h e)$ are made of the association of a dark $(d s)$ and a bright $(b s)$ strip. New herringbone units appear at dislocations $(d i)$. The wavy black dashed line represents a past growth surface and indicates the polarity of the structure. (c) Internal structure of the herringbone: each strip is made of elongated spindles ( $s p$ ). Spindles in two adjacent strips in a herringbone unit form an angle of $\sim 45^{\circ}$ (see also b). Black arrows indicate both the elongation of the spindles and the orientation of the $c$ axes of calcite crystals. (d) SEM observation of spindles. Differences in gray levels are due to orientation contrast; the limit between two adjacent spindles corresponds to a change in crystallographic orientations. Yellow lines and black arrows underline the $c$ axis orientations in different spindles. Note the drastic change of orientations on both sides of the herringbone axis shown as a heavy black line. The white dashed lines are traces of growth rings; they are orthogonal to crystallographic orientations. (e) Internal structure of the spindles (orientation contrast SEM image). Spindles are made of lozenges (lo). These lozenges are reminiscent of sections of calcite scalenohedra and are separated by dark zones indicative of porosity ( $p o$ ). Each lozenge shows elongated dark/bright bands ( $b a$ ) corresponding to crystalline fibers. Black arrows indicate the $c$ axis orientations. (f) Crystalline fibers $(f i)$ observed under the SEM (secondary electrons). Fibers are made of a piling of submicrometer units $(\mathrm{sm})$. (g) A crystalline fiber in a focused ion beam foil observed under the TEM, dark-field image. (h) Submicrometer units under the TEM in a focused ion beam foil. Variations in gray levels are due to variations in crystallographic orientations. The darkest zones between the crystals are indicative of porosity ( $p o$ ). (i) HRTEM image showing lattice fringes and the internal structure of submicrometer units composed of 2-5 nm similarly oriented nanodomains. Variations in adsorption contrast indicate the presence of nanopores $(n p)$. Some characteristic interplane distances are indicated in nanometers. Inside frames in $\mathbf{b}, \mathbf{c}, \mathbf{d}, \mathbf{e}, \mathbf{f}$, and $\mathbf{h}$ are drawn for relative scale purpose and do not represent exact locations of enlargements. 

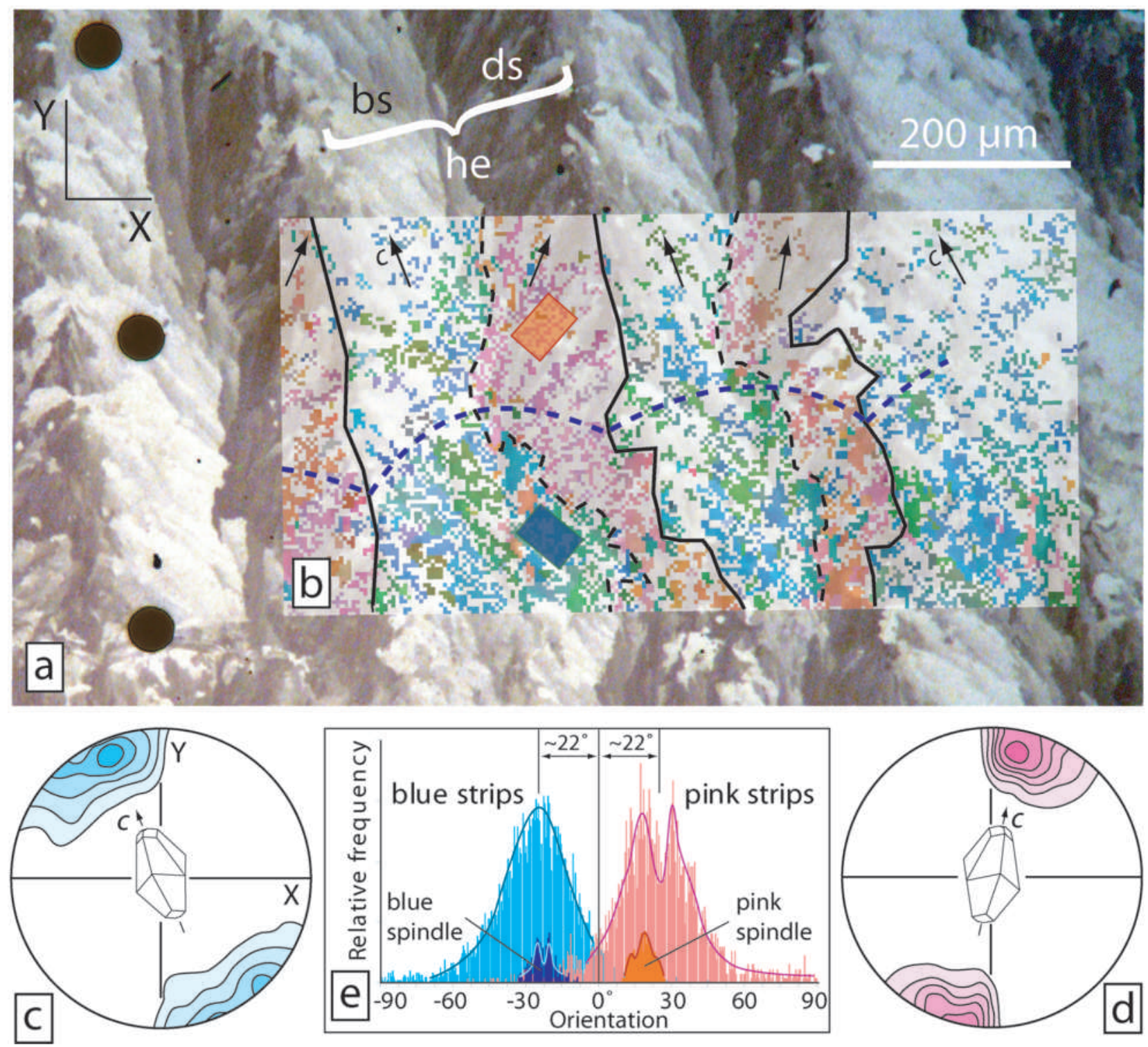

FIGURE 2. EBSD study. (a) Reflected light microphotograph of a section of red coral skeleton normal to the axis (compare to Fig. 1a); $b s, d s$, and he as in Figure 1b. (b) Electron back-scattered diffraction orientation map: $200 \times 100$ grid with a $4 \mu \mathrm{m}$ interval between points, spatial resolution $\sim 200 \mathrm{~nm}$. For relative scale and polarity purpose, the wavy dark-blue dashed line represents a fossil growth surface. The black arrows correspond to the orientation of the $c$ axes of the calcite crystals in the different strips. The dark-blue and dark-orange rectangles are the selected areas for the blue and pink spindle histograms in e. (c and d) Pole figures of the $\{0001\}$ planes in the blue and pink strips. Density plot, stereographic projection, half width $10^{\circ}$, cluster size $5^{\circ}$, contour line interval 2.5. (e) Histograms of crystallographic orientation of the $c$ axes in the XY plane of crystals in the blue and pink strips (entire map) and in a blue and pink spindle (selected areas shown in b).

Prior et al. 1999). SEM and TEM observations (Figs. 1f $[f i]$ and $\lg [f i])$, respectively, indicate the existence of crystalline fibers (see also Vielzeuf et al. 2008) whose dimensions (ca. $1 \mu \mathrm{m}$ wide, ca. $10 \mu \mathrm{m}$ long) are identical to the elongated bands observed in the lozenges. Thus, we infer that the OC bands in the lozenges correspond to crystalline fibers.

\section{Submicrometer units $\left(10^{-7} \mathrm{~m}\right)$}

In a previous SEM and TEM study, Vielzeuf et al. (2008) showed that the micrometer-scale crystalline fibers in the red coral do not correspond to single crystals, but are themselves made of the piling of $200-500 \mathrm{~nm}$ units (Fig. 1f [ sm]). This con- clusion is consistent with atomic force microscopy observations (Dauphin 2006). These submicrometer units are clearly identified on focused ion beam foils observed with a TEM (Fig. 1h [ $5 \mathrm{sm}]$ ). In the dark-field images, the boundaries of the submicrometer units are commonly marked by thin black porous spaces (Fig. $1 \mathrm{~h}$ $[p o])$. Differences in diffraction contrast from one submicrometer unit to the other (Figs. 1g and $1 \mathrm{~h}$ ) can be ascribed to slight crystallographic misorientations.

\section{Nanodomains $\left(10^{-8}-10^{-9} \mathrm{~m}\right)$}

TEM electron diffraction patterns on selected areas in the submicrometer units, such as the one shown as an inset in 


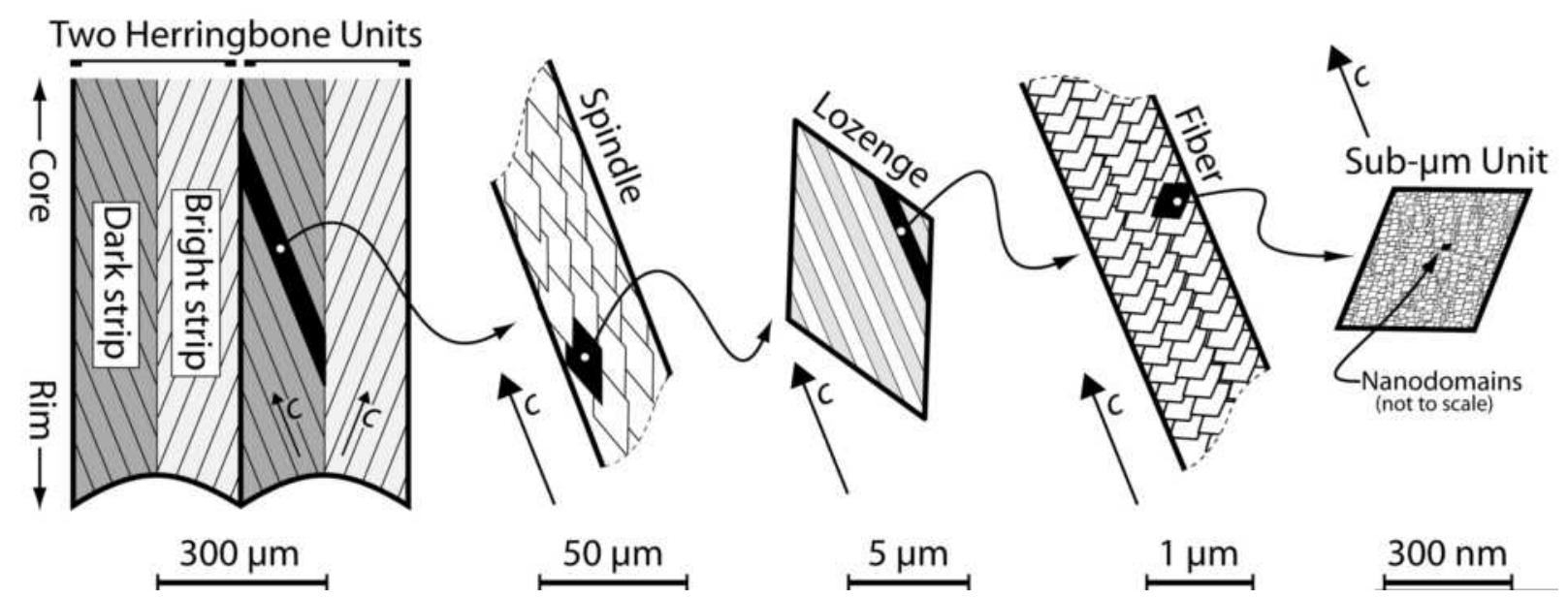

FIGURE 3. Summary and conceptual interpretation of seven levels of crystallographic hierarchy in the skeleton of Corallium rubrum. The macroscopic radial arrangement of herringbone units (as in Fig. 1a) representing an eighth level is not shown here. For clarity, some angles and lengths are not to scale. Note the preservation of crystallographic order ( $c$ axis) over long distance. Possible slight misorientations between the constituting units are not shown.

Figure 1i, indicate that each unit diffracts as a single crystal. However, TEM images show that the submicrometer units are not single crystal, but are made of an assemblage of $2-5 \mathrm{~nm}$ nanodomains (Vielzeuf et al. 2008). Variations in absorption contrast and the presence of free crystalline surfaces suggest the presence of an intimate network of nanopores (Vielzeuf et al. 2008). High-resolution TEM imaging (Fig. 1i) confirms the presence of nanograins. Some of them display a rhombohedral shape. Most importantly, the parallelism of lattice fringes across adjacent domains demonstrates that the crystallographic axes of all domains are nearly parallel.

\section{DISCUSSION AND IMPLICATIONS}

Figure 3 summarizes the different levels of crystallographic arrangement in the red coral skeleton. From this hierarchy of crystallographic structures, a simple pattern emerges: imperfectly similarly oriented (ISO) nanograins combine into submicrometer units; the ISO submicrometer units combine into fibers; ISO fibers make up the lozenge units; ISO lozenges are arranged into spindles; the ISO spindles comprise the millimetric strips; the strips joined side by side form herringbone units; and finally, the juxtaposition of herringbone units plus the addition of new units at macroscopic dislocations lead to the radiating structure of the red coral skeleton. Each entity is composed of modules and is at the same time a modular part of a larger module with remarkable similarity of crystallographic orientations. Histograms of crystallographic orientation of $c$ axes (Fig. 4) can be used to present a Russian nesting doll-like organization that spans over seven orders of magnitude. We have already seen that the histogram of orientation in the millimeter-scale strips (Fig. 2e) is the envelope of the histograms of the spindles that constitute the strip. In turn, the spindle histogram is necessarily the envelope of the histograms of the lozenges that constitute the spindles, and so on. Observations with different analytical techniques from the reflected light microscope to the TEM suggest that the degree of misorientation decreases with the decreasing size of the crystallographic modules. This is demonstrated by EBSD measurements for the strip and the spindle units. Our attempts to quantify the degree of misorientation in the smallest units by doing EBSD maps with a better spatial resolution $(0.2 \mu \mathrm{m}$ spacing between points) failed, in part as a result of beam damage of the sample. However, qualitative observations of the smallest units as described earlier are in agreement with the interpretation of increasing order with decreasing size of the modules. Thus, if our interpretation is correct, the red coral skeleton shows a progressive transition from imperfect to almost perfect crystalline domains down through a hierarchical crystallographic structure. This multilevel modular arrangement preserves long-range crystallographic ordering (though imperfect), scattering properties close to single crystals and, at the same time, it allows the pres-

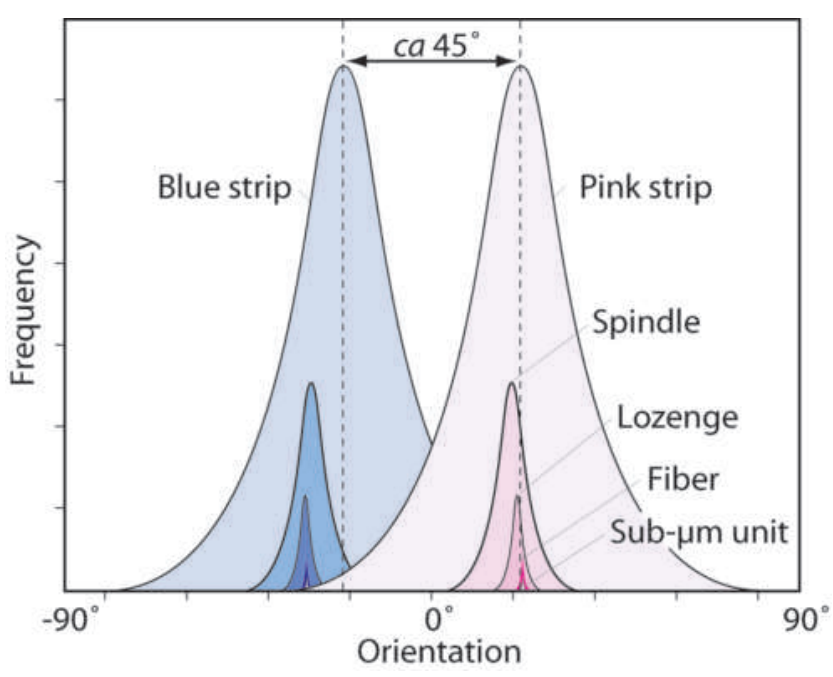

FIGURE 4. Modular organization illustrated through a schematic composite histogram of crystallographic orientations. Each unit is the external envelope of various sub-units and at the same time a sub-unit of a larger envelope. The degree of misorientation in the sub-unit is lower than in the unit. Note that the distribution in the blue and pink strip overlap. Not to scale. 
ence of porosity and crystal interfaces at all scales.

A "mesocrystal" is a recent concept defined as a superstructure made of almost perfectly aligned crystalline particles (Cölfen and Antonietti 2005; Meldrum and Cölfen 2008). This concept arose from observations of natural and synthetic materials (Penn and Banfield 1998, 1999). In these examples, primary particles aligned, docked, and fused to form oriented chains by a mechanism called "oriented attachment" (Penn and Banfield 1998). This mechanism was an elaboration of the concept of "particle aggregation" developed for colloids (Bailey et al. 1993; Privman et al. 1999); it applies in biomineralogy as shown by experiments in the $\mathrm{CaCO}_{3}$ system (Cölfen and Antonietti 2005; Imai et al. 2006; Oaki et al. 2007). An important point is that although they are made of distinct nanoparticles, mesocrystals display scattering properties of single crystals (Cölfen and Antonietti 2005). Most experimental studies related to mesocrystals describe a single level of organization, i.e., nanograins assembling into a mesocrystal. However, recently, in a study dealing with the growth of calcite in an organic gel matrix, Oaki et al. (2007) observed structures made of a hierarchy of nanocrystals, submicrometric and micrometric units, each exhibiting rhombohedral habits. They concluded that the resultant calcite could be regarded as a hierarchical self-similar structure.

Our observations are consistent with most aspects of mesocrystals as described in these references, and thus we infer that each of the hierarchical levels we have observed in the red coral skeleton would be appropriately described as mesocrystalline. However, some observations presented here are new and may help refining the concept of mesocrystals in biomineral systems. For example, contrary to previous examples of mesocrystals but in agreement with the experimental observations of Oaki et al. (2007), the red coral skeleton displays not a single stage but rather a multilevel crystallographic hierarchy. This is a new feature of natural mesocrystals. Further studies will be required to determine if, as we expect, this is a general feature of biominerals.

Mesocrystals have been viewed as examples of crystallization that does not proceed through ion-by-ion attachment but rather by attachment of modular building blocks (Cölfen and Antonietti 2005). Our observations only describe a hierarchical organization and not necessarily a crystallization mechanism. Given the way in which a red coral skeleton grows (i.e., via layer-by-layer addition of particles at the surface, as shown by chemical patterns and the presence of annual growth rings (Marschal et al. 2004; Vielzeuf et al. 2008), it seems likely that only the smallest hierarchical scales may have been assembled by the addition of separately built modules. In the case of the red coral, we have a modular organization but not a modular construction [see Baldwin and Clark (1997) for an interesting analogy with modularity in the design of complex engineering systems]. A major difference between inorganic mineral structures (e.g., rocks) and biomineral structures (e.g., skeletons), is that the latter are evolved by organisms to fulfill particular functions. The skeleton of the red coral must respond to competing demands: anchor the colony on a rocky sea floor; support and grip the living tissues; allow the growth of the organism; achieve mechanical strength against sea currents; and adapt its shape in a way that favors both the access of the polyps to the nutrients and the removal of metabolic waste in the sea water. The modular crystallographic organization in the red coral and its ability to achieve complex morphologies overcome the poor mechanical properties of calcite. For instance, microprotuberances, the morphological surface expression of the crystallographic spindles as discussed earlier, end up acting as self-blocking structures between growth rings in the skeleton (Vielzeuf et al. 2008). Thus, in the red coral, morphologically complex, mechanically resistant structures result from a construction scheme ruled in great part by crystallographic principles.

Colonies of red coral display a wide range of morphologies indicating that they are able to adapt to surrounding hydrodynamic motion. We noted above that the similarity of crystallographic orientation between different modules in each level is imperfect. Such misorientations may not be viewed as "imperfections" but rather as degrees of freedom that, together with the polydispersity of the modules and the presence of interfaces at all levels, allow morphological adjustments. The precise nature of the interfaces between the crystalline units is not yet known. However, Vielzeuf et al. (2008) noted the presence of porosity and organic matter at all scales, indicating that the red coral skeleton is a composite organic/inorganic material. Thus, both inorganic/inorganic and organic/inorganic types of interfaces are possible. It has been suggested that the formation of biogenic calcium carbonates is directed by an ordered template of macromolecules (Cuif and Dauphin 2005; Pouget et al. 2009). Whether the hierarchical modular organization presented here is also directed by a hierarchical organization of macromolecules remains to be determined.

In nature, the existence of an amorphous phase that could be "molded into any shape" has been put forward as a key factor of morphological control in biominerals (Weiner et al. 2005). We do not challenge the existence and the importance of an amorphous phase as a precursor in crystalline biominerals, but we doubt that it plays a major role in the final morphology of the red coral. Instead, we consider that the presence of interfaces at all scales is a key property of biominerals to achieve all kinds of shapes and adapt to the environment. How amorphous precursor phase may influence or relate to the multiscale interface model that we propose here is not yet known.

Developing nanomaterials with controlled hierarchical structures, crystalline morphology, orientation, and surface architecture remains a challenge in materials science, and there is still much to learn from biomineral archetypes (Cölfen 2003). The multiscale interface model presented here may prove useful to develop new strategies to design complex-shaped threedimensional crystalline synthetic materials.

\section{ACKNOWLEDGMENTS}

This work has been supported by Centre National de la Recherche Scientifique (CNRS), Institut National des Sciences de l'Univers (INSU) through grants ECLIPSE 2005, INTERRVIE 2009, and by Centre Interdisciplinaire de Nanoscience de Marseille (CINaM) through internal grants to D.V. We thank C. Henry for his support, Chi Ma for his assistance with EBSD and OC imaging at Caltech, and C. Vanni, W. Saikali, C. Dominici, and T. Neisius for their help with the FIB foil preparation and observation at CP2M-Marseille. We made the TEM observations at the INSU TEM facility at Marseille. We thank S. Nitsche and D. Chaudanson for their supervision during the sessions. One of us (D.V.) is grateful for a Caltech financial support to this project. Reviews by three anonymous reviewers as well as editorial handling by Nita Sahai are gratefully acknowledged.

\section{REFERENCES CITED}

Bailey, J.K., Brinker, C.J., and Mecartney, M.L. (1993) Growth mechanisms of ironoxide particles of differing morphologies from the forced hydrolysis of ferric- 
chloride solutions. Journal of Colloid and Interface Science, 157, 1-13.

Baldwin, C.Y. and Clark, K.B. (1997) Managing in an age of modularity. Harvard Business Review, 75, 84-93.

Cölfen, H. (2003) Precipitation of carbonates: recent progress in controlled production of complex shapes. Current Opinion in Colloid and Interface Science, 8, 23-31.

Cölfen, H. and Antonietti, M. (2005) Mesocrystals: Inorganic superstructures made by highly parallel crystallization and controlled alignment. Angewandte Chemie-International Edition, 44, 5576-5591.

Cuif, J.P. and Dauphin, Y. (2005) The Environment Recording Unit in coral skeletons - a synthesis of structural and chemical evidences for a biochemically driven, stepping-growth process in fibres. Biogeosciences, 2, 61-73.

Dauphin, Y. (2006) Mineralizing matrices in the skeletal axes of two Corallium species (Alcyonacea). Comparative Biochemistry and Physiology a-Molecular and Integrative Physiology, 145, 54-64.

Grillo, M.C., Goldberg, W.M., and Allemand, D. (1993) Skeleton and sclerite formation in the precious red coral Corallium rubrum. Marine Biology, 117, $119-128$.

Grimm, V., Revilla, E., Berger, U., Jeltsch, F., Mooij, W.M., Railsback, S.F., Thulke, H.H., Weiner, J., Wiegand, T., and DeAngelis, D.L. (2005) Patternoriented modeling of agent-based complex systems: Lessons from ecology. Science, 310, 987-991.

Imai, H., Oaki, Y., and Kotachi, A. (2006) A biomimetic approach for hierarchically structured inorganic crystals through self-organization. Bulletin of the Chemical Society of Japan, 79, 1834-1851.

Lowenstam, H.A. and Weiner, S. (1989) On Biomineralization. Oxford University Press, New York.

Mann, S. (2001) Biomineralization: Principles and concepts in bioinorganic materials chemistry. Oxford University Press, New York.

Marschal, C., Garrabou, J., Harmelin, J.G., and Pichon, M. (2004) A new method for measuring growth and age in the precious red coral Corallium rubrum (L.). Coral Reefs, 23, 423-432.

Meldrum, F.C. and Cölfen, H. (2008) Controlling mineral morphologies and structures in biological and synthetic systems. Chemical Reviews, 108, 4332-4432.

Oaki, Y., Hayashi, S., and Imai, H. (2007) A hierarchical self-similar structure of oriented calcite with association of an agar gel matrix: inheritance of crystal habit from nanoscale. Chemical Communications, 2841-2843.

Pecher, J., Guenoun, P., and Chevallard, C. (2009) Crystalline calcium carbonate thin film formation through interfacial growth and crystallization of amorphous microdomains. Crystal Growth and Design, 9, 1306-1311.

Penn, R.L. and Banfield, J.F. (1998) Imperfect oriented attachment: Dislocation generation in defect-free nanocrystals. Science, 281, 969-971.

(1999) Morphology development and crystal growth in nanocrystalline aggregates under hydrothermal conditions: Insights from titania. Geochimica et Cosmochimica Acta, 63, 1549-1557.

Pouget, E.M., Bomans, P.H.H., Goos, J., Frederik, P.M., de With, G., and Sommerdijk, N. (2009) The initial stages of template-controlled $\mathrm{CaCO}_{3}$ formation revealed by cryo-TEM. Science, 323, 1555-1458.

Prior, D.J., Boyle, A.P., Brenker, F., Cheadle, M.C., Day, A., Lopez, G., Peruzzo, L., Potts, G.J., Reddy, S., Spiess, R., Timms, N.E., Trimby, P., Wheeler, J., and Zetterstrom, L. (1999) The application of electron backscatter diffraction and orientation contrast imaging in the SEM to textural problems in rocks. American Mineralogist, 84, 1741-1759.

Privman, V., Goia, D.V., Park, J., and Matijevic, E. (1999) Mechanism of formation of monodispersed colloids by aggregation of nanosize precursors. Journal of Colloid and Interface Science, 213, 36-45.

Towe, K.M. (2006) Sea urchins as crystallographers. Science, 311, 1554-1555.

Vielzeuf, D., Garrabou, J., Baronnet, A., Grauby, and Marschal, C. (2008) Nano to macroscale biomineral architecture of red coral (Corallium rubrum). American Mineralogist, 93, 1799-1815.

Weiner, S., Sagi, I., and Addadi, L. (2005) Choosing the crystallization path less traveled. Science, 309, 1027-1028.

MANUSCRIPT RECEIVED APRIL 10, 2009

MANUSCRIPT ACCEPTED OCTOBER 13, 2009

MANUSCRIPT HANDLED BY NITA SAHAI 